\title{
Structure Analysis of a Sugar-moiety Chimera of EmaA, a Collagen Adhesin of a Gram-negative Bacterial Pathogen
}

\author{
Gaoyan Tang-Siegel ${ }^{1}$, Claire J. Brooks ${ }^{1}$, Michael Radermacher ${ }^{1}$, Keith P. Mintz ${ }^{2}$, and Teresa Ruiz ${ }^{1}$ \\ ${ }^{1}$ Department of Molecular Physiology \& Biophysics, University of Vermont, Burlington, VT \\ ${ }^{2}$ Department of Microbiology \& Molecular Genetics, University of Vermont, Burlington, VT
}

The gram-negative non-motile bacterium, Aggregatibacter actinomycetemcomitans is an oropharyngeal colonizer. A. actinomycetemcomitans causes aggressive periodontitis and systemic infections, including endocarditis and pneumonia. Multiple virulence factors are involved in the infective process, and EmaA (extracellular matrix adhesin A) is one of the important virulence determinants in the early stage of infection. EmaA is a trimeric autotransporter adhesin, composed of three $202 \mathrm{kDa}$ monomers, and forms antenna-like appendages extending more than $150 \mathrm{~nm}$ from the bacterial surface [1]. The functional Nterminus, encompasses approximately $30 \mathrm{~nm}$ from the distal end, and comprises three subdomains (SI, SII and SIII), with a linker region between subdomains SII and SIII [2]. EmaAs are hypothesized to be glycosylated by a novel post-translational mechanism. This mechanism shares the same enzyme that ligates the O-polysaccharide (O-PS) sugars to the lipopolysaccharide [3]. Seven different serotypes associated with $A$. actinomycetemcomitans have been identified based on different O-PS sugars. Accordingly, the sugars present in the EmaA of each serotype are different. The O-PS of serotype a strains is composed of repeated disaccharide units of talose, while the O-PS of serotype b strains consists of repeated rhamnose-fucose-acetylgalactosamine trisaccharides. Glycosylation, as well as the primary sequence, are important for the function and stability of the EmaA adhesin [4]. Therefore, 3D structures of EmaA (the serotype $b$ sequence) modified with the sugars from a serotype a strain (talose), a sugar-moiety chimera, were reconstructed for better understanding the functional role of glycosylation in this adhesin.

Electron tomography analysis was performed to resolve the 3D structure of a sugar-moiety chimera of EmaA. A plasmid carrying the full-length serotype b emaA sequence was transformed into a serotype a $e m a A^{-}$mutant strain (a-emaA $)$to generate a new strain a-emaA $/ \mathrm{b}-e m a A^{+}$. The sugar-moiety chimera EmaA (a-EmaA) expressed in this strain was compared to the wild type b-EmaA expressed in a serotype $\mathrm{b}$ strain. The collagen binding activity of the a-emaA $/ \mathrm{b}-\mathrm{emaA} A^{+}$strain was determined using an enzymelinked absorbent assay (ELISA) [3,4]. Electron microscopy was performed on deep negatively stained whole-mount bacterial preparations [5]. Bacteria were grown in broth and collected by centrifugation at $\times 3,000 \mathrm{~g}$ for $1 \mathrm{~min}$ at $4^{\circ} \mathrm{C}$, A $5 \mu$ aliquot of bacterial suspension was placed onto carbon-coated grids pretreated with low molecular weight polylysine $(\sim 3 \mathrm{kDa})$ and colloidal gold, and negatively stained with Nano W (Nanoprobes, NY). Grids were imaged at $100 \mathrm{kV}$ with a Tecnai 12 electron microscope (FEI, OR) Tomographic tilt series were acquired within a $\pm 64^{\circ}$ angular range at $2^{\circ}$ angular intervals. Tomographic reconstructions of the whole imaged area were calculated using IMOD. For each selected EmaA from the tomogram, a tilt series of subprojections was extracted and subvolumes were calculated with the long-axis of the adhesin approximately parallel to the Y-axis of the subvolume using EMIRA [6]. The a-EmaA reconstructions were aligned to the reference b-EmaA using Radon transform algorithms implemented in EMIRA, and visualized using Chimera [7]. Probabilistic Principal Component Analysis (PPCA-EM) implemented EMIRA was used to assess differences between all aEmaA subvolumes [6,8]. The 3D reconstructions were further grouped based on similarities and averages were calculated for each group to use as references in a multireference alignment step. After 
several iterations of multivariate statistic analysis and multireference alignment, an average for each group was calculated and visualized in Chimera.

The a-EmaA is functional, as demonstrated by a 3-fold increased binding to collagen $\mathrm{V}$ of the a-emaA $/ \mathrm{b}-\mathrm{emaA}^{+}$strain when compared to the a-emaA ${ }^{-}$mutant strain by ELISA $(P<0.05)$, which is different from the EmaAs of glycosylation deficient strains [4]. Moreover, the overall appearance of the a-EmaA chimera is similar to the wild type b-EmaA. In both strains, EmaA adhesins extend away from the bacterial surface (Fig. 1a). In contrast, the EmaAs from glycosylation deficient strains are hugging the bacterial surface [4]. Further analysis of the 3D structures of the functional domain demonstrated alterations in the a-EmaA sugar-moiety chimera, when compared to the wild type b-EmaA. Five subgroups were identified based on structure similarities: GI, GII, GIII, GIV and GV (Fig. 1b). The structural changes of the a-EmaA appear to be the consequence of different sugar moieties, since the protein sequence is the same. Moreover, we are further investigating the amount and nature of the conjugated sugars.

\section{References:}

[1] Ruiz T., Lenox C., Radermacher M., and Mintz K.P., Infect. Immun. 74 (2006), p. 6163-6170.

[2] Yu, C., Mintz, K.P., Ruiz, T., J. Bacteriol. 191 (2009), p.6253-6261.

[3] Tang G., and KP Mintz, J. Bacteriol. 192 (2010), p. 1395-1404.

[4] Tang, G., Ruiz, T., Mintz, K.P., Infect. Immun. 80 (2012), p.2868-2877.

[5] Azari, F., Radermacher, M., Mintz, K.P., Ruiz, T., J. Struct. Biol. 177 (2011), p.439-446.

[6] Radermacher, M., Microscopy \& Microanalysis, 19 (2013), Supplement S2 p.762-763.

[7] Petterson, E.F., Goddard, T.D., et al., J. Comput. Chem. 25 (2004), p.1605-1612. [

[8] Yu, L., Snapp, R.R., Ruiz, T., and Radermacher, M. (2010). J Struct Biol 171, 18.

[9] This work was supported by NIH grant DE024554, (T.R. \& K.P.M) and GM078202 (M.R.).

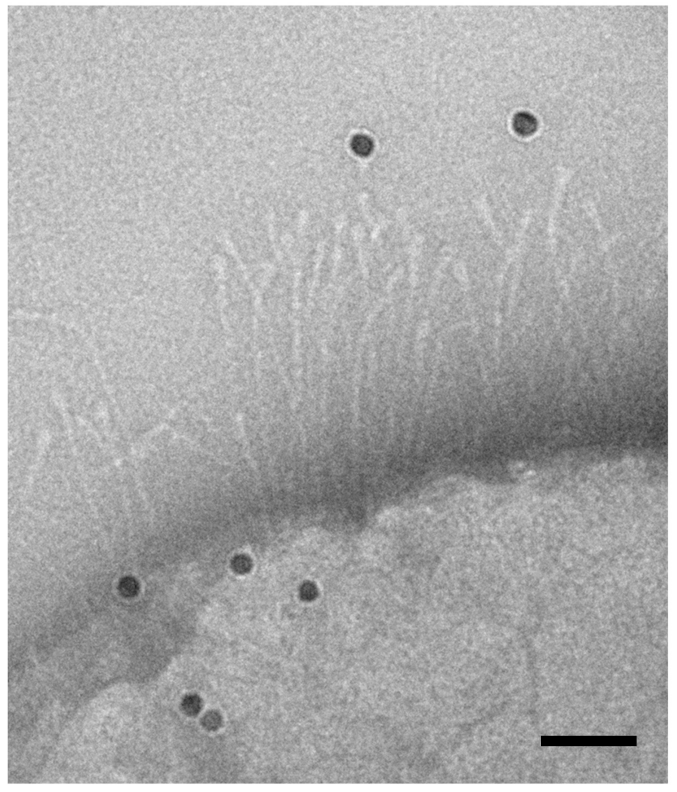

(a)

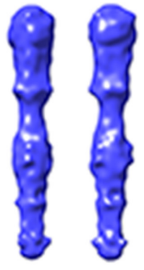

b-EmaA

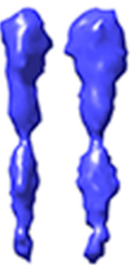

GIII

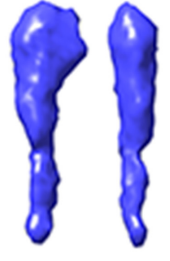

GI

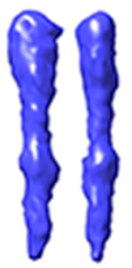

GIV

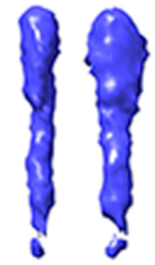

GII

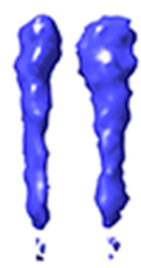

GV

Figure 1. Structural analysis of the sugar-moiety chimera a-EmaA functional domain. (a) Electron micrograph, $0^{\circ}$-projection. Bar: $30 \mathrm{~nm}$ (b) Surface representation of five subgroups of a-EmaA (GI-GV) versus the wild type b-EmaA. Length represented: $30 \mathrm{~nm}$. 